\title{
Root-exuded malic acid versus chlorophyll fluorescence parameters in four plant species under different phosphorus levels
}

Y. Y. Wu ${ }^{1,2,3 *}$ and K. Zhao ${ }^{1,2}$

${ }^{1}$ Key Laboratory of Modern Agricultural Equipment and Technology, Ministry of Education,China. ${ }^{2}$ Institute of Agricultural Engineering, Jiangsu University, Zhenjiang, 212013, P. R. China. ${ }^{3}$ State Key Laboratory of Environmental Geochemistry, Institute of Geochemistry, Chinese Academy of Sciences, Guiyang, 550002, P. R. China. *Corresponding author: wuyanyou@mail.gyig. ac.cn

\begin{abstract}
The amount of root-exuded malic acid and chlorophyll fluorescence parameters (the minimum chlorophyll fluorescence; the maximum quantum yield of photosystem II) in four plant species (Broussonetia papyrifera, Morus alba, Orychophragmus violaceus and Brassica napus) at different phosphorus levels was studied. A linear equation presents the minimum chlorophyll fluorescence (Fo) or the maximum quantum yield of photosystem II $(\mathrm{Fv} / \mathrm{Fm})$ and the amount of root-exuded malic acid. Broussonetia papyrifera and Orychophragmus violaceus easily adapted to a low-phosphorus environment. A low-cost method was used for assessing plant adaptability to a lowphosphorus environment when no chlorophyll fluorescence instrument was available.
\end{abstract}

Keywords: Adaptability, chlorophyll fluorescence parameters, malic acid, low phosphorus environment

\section{Introduction}

Malic acid is one of the most common low molecular weight organic acids in root exudates, root-exuded organic acids are important mechanism in response to environment stress (Jones, 1998). Recently, many studies revealed that the amount of root-exuded organic acids increases under nutrient stress to increased nutrient availability (Dakora and Phillips, 2002).
Phosphorus $(\mathrm{P})$ is an important factor that can limit plant growth and development; the amount of available P directly determines plant growth condition (Broadley et al., 2002). Many studies indicate that the low molecular weight organic acids significantly increase under P deficiency. These organic acids complex with metallic cations to form organ-metallic complexes to overcome P deficiency (Hoffland et al., 2006). 
Chlorophyll fluorescence kinetics technology is called for fast and undamaged probe for studying plant photosynthetic characteristics. This technology is applied in plant stress physiology, pollution determination, remote sensing and others (Ogren, 1990 ; Qiu and Liu, 2000; Meroni, 2009 ). It is an ideal method for the study of plant physiology, such as nutrients stress (Lu and Zhang, 2000; Lippemeier et al., 2003).

This study determined the amount of root-exude malic acid in different plant species at P-sufficient, P-low and P-deficient levels. The chlorophyll fluorescence parameters were examined. A new method to evaluate plants adaptability to a low-P environment through the amount of root-exuded malic acid was proposed.

\section{Materials and Methods}

\subsection{Solution culture experiment}

Seeds of the four plant species (two Moraceae plants: Broussonetia papyrifera, B. papyrifera; Morus alba, M. alba; two Cruciferous plants: Orychophragmus violaceus, O. violaceus; Brassica napus, B. napus) were germinated in plastic pots for 15 days. The seedlings were washed with distilled water and carefully transferred to plastic tubes filled with Hoagland nutrient solutions (Hoagland and Arnon, 1950). The $\mathrm{pH}$ was adjusted to 8.0 by KOH. After 30 days, the plants were transferred to a modified Hoagland nutrient solution with different $\mathrm{P}$ levels for treatment, as modified by $\mathrm{KH}_{2} \mathrm{PO}_{4}: 0.5 \mathrm{mM} \mathrm{P}$ (P-sufficient), $0.1 \mathrm{mM} \mathrm{P}$ (P-low), and without $\mathrm{P}$ (P-deficient). Root exudates were collected after 30 days treatment durations.

\subsection{Collection, separation, purification and analysis of root exudates}

Collection, separation, purification of malic acid in root exudates is the method of Wang (2007). The amount of the malic acid was analyzed by reversed-phase highperformance liquid chromatography (Cawthray, 2003).

\subsection{Chlorophyll fluorescence measurements}

Chlorophyll fluorescence is measured by an imaging pulse-amplitude-modulated fluorometer (Imaging PAM; Heinz Walz, Effeltrich, Germany), which applied the same array of blue light-emitting diodes (LEDs) (peakwavelength, $470 \mathrm{~nm}$ ) for fluorescence excitation, actinic illumination, and saturating light pulses. Plants were dark-adapted for $30 \mathrm{~min}$ prior to measurement, and the upper middle fully expanded leaves of four plant species were chosen and measured (Fu et al., 2012). The minimum Chlorophyll fluorescence (Fo) was determined using a measuring beam, whereas the maximum Chlorophyll fluorescence (Fm) was recorded after exposure to a $0.8 \mathrm{~s}$ saturating light pulse $\left(6000 \mu \mathrm{mol} \cdot \mathrm{m}^{-2} \cdot \mathrm{s}^{-1}\right)$. The maximum quantum yield of PSII $(\mathrm{Fv} / \mathrm{Fm})$ was calculated as $(\mathrm{Fm}-\mathrm{Fo}) / \mathrm{Fm}$.

\section{Results}

\subsection{Amount of root-exuded malic acid in four plant species at different $P$ levels}

As shown in Figure 1, the amount of malic acid in four plant species root exudates increased as the $\mathrm{P}$ level decreased after treatment of 30 days. The amount of root-exuded malic acid at the P-deficient level is significantly higher than that at the P-sufficient and P-low levels. For the two Moraceae plants, the amount of B.papyrifera root-exuded malic acid was remarkably higher than that in M.alba in all three $\mathrm{P}$ levels $(p<0.01)$. The amount of malic acid in B.papyrifera root exudates was 1-folder greater than that in M.alba. Root-exuded malic acid increased up to $60 \%$ for B.papyrifera and $86 \%$ for M.alba, with the $\mathrm{P}$-sufficient level sample for the same species acting as control. For the two Cruciferous plants, malic acid in O.violaceus was higher than that in B.napus. Increased in root-exuded malic acid was $79 \%$ for $O$.violaceus and $81 \%$ for B.napus, with the P-sufficient level sample for the same species acting as control. There was no significant difference in the two Cruciferous plants at 
the P-sufficient and P-low levels, while there was a difference at the P-deficient level $(p<0.05)$.

\subsection{Variation of chlorophyll fluorescence parameters}

Figure 2 showed the variation of chlorophyll fluorescence parameters $\mathrm{Fv} / \mathrm{Fm}$ and $\mathrm{Fo}$ at the three $\mathrm{P}$ levels. Fv / Fm decreased, Fo increased as the P level decreased in four plant species. For the two Moraceae plants, there was no difference at the P-sufficient level and P-low level; Fv / Fm was greater in B.papyrifera than in M.alba at the P-deficient level $(p<0.05)$. There was no significant difference in Fo in the two Moraceae plants at all three P levels. For the two Cruciferous plants, there was no difference in $\mathrm{Fv} / \mathrm{Fm}$ at all three $\mathrm{P}$ levels, Fo was greater in O.violaceus than in B.napus at the P-low level and P-deficient level $(p<0.05)$

\subsection{Amount of root-exuded malic acid versus chlorophyll fluorescence parameters}

As shown in Table 1, a linear equation can simulate the relationship between the amount of root-exuded malic acid and chlorophyll fluorescence parameters (Fv/ $\mathrm{Fm}, \mathrm{Fo})$. The determination coefficient $\left(\mathrm{R}^{2}\right)$ ranged from 0.785 to $0.992, \mathrm{R}^{2}$ of B.papyrifera, O.violaceus, B.napus was greater than 0.99 , except M.alba.

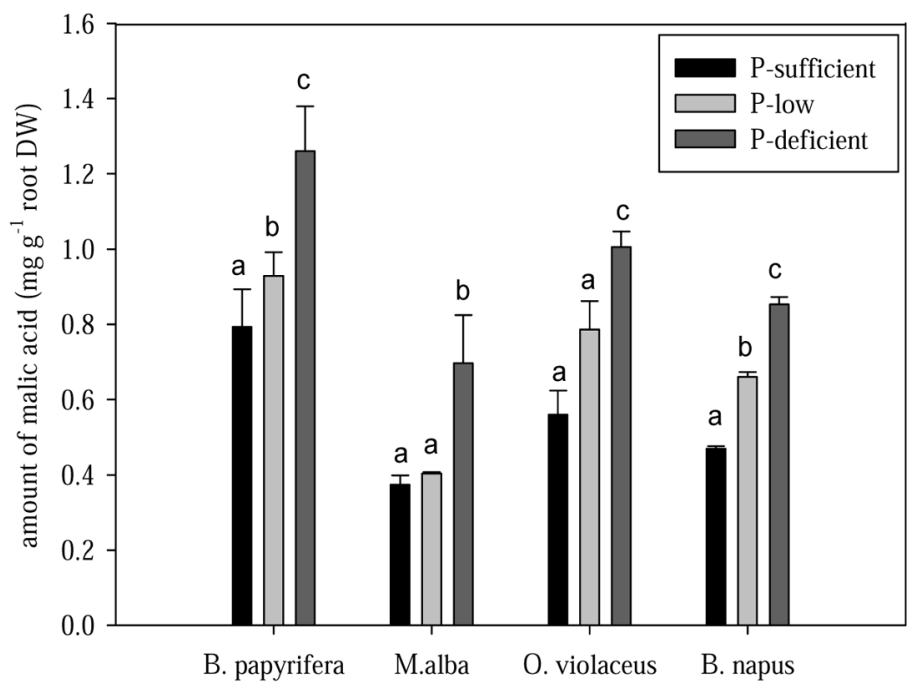

Figure 1. The amount of root-exuded malic acid in four plants under different phosphorus levels. Note: The histogram with bars indicated the means \pm SE. Different letters indicate significant differences at the $p<0.05$ level, according to one-way ANOVA and t-test. 

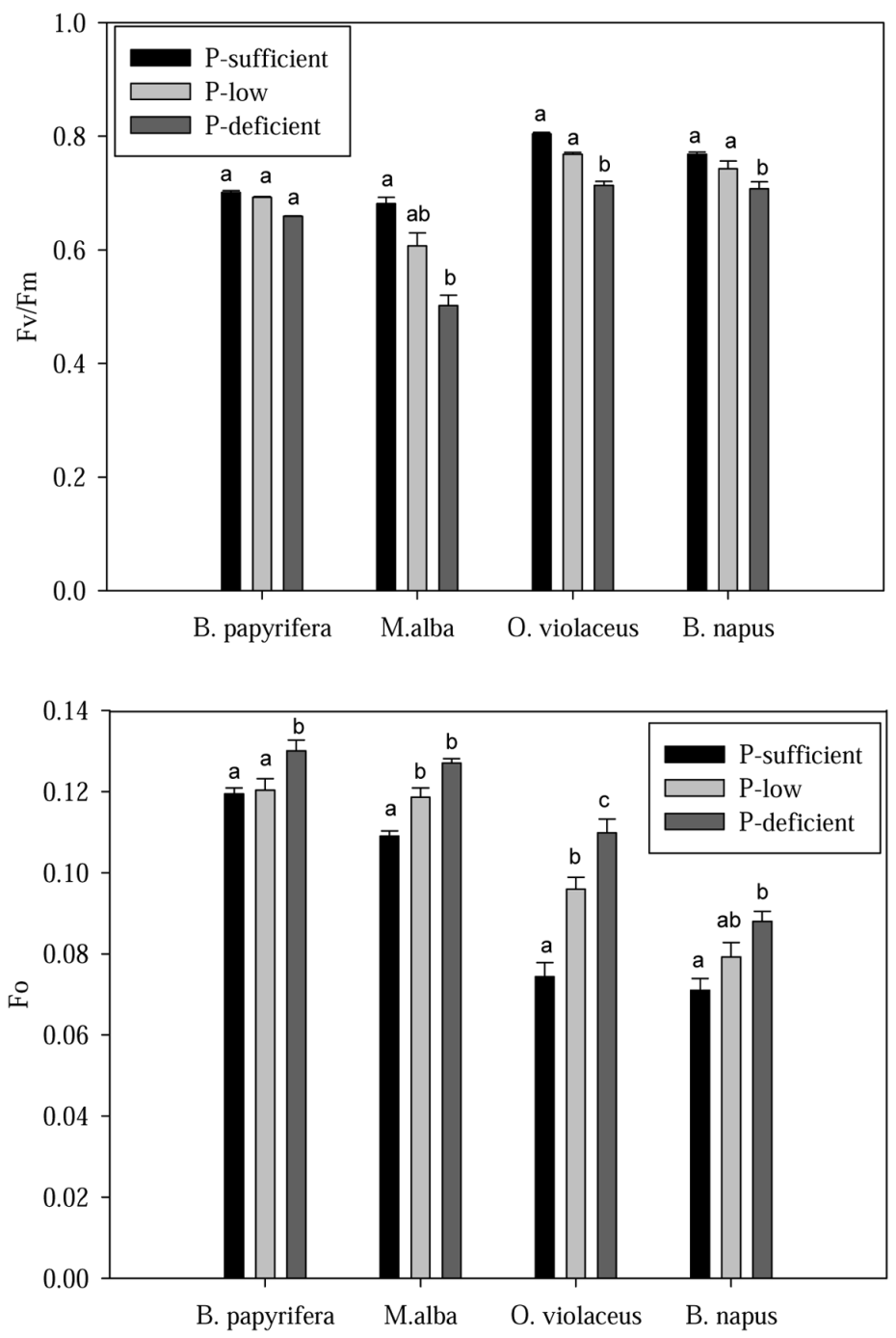

Figure 2. The effect of different phosphorus levels on chlorophyll fluorescence parameters of four plants. Note: The histogram with bars indicated the means \pm SE. Different letters indicate significant differences at the $p<0.05$ level, according to one-way ANOVA and t-test. 
Table 1. Regression equations between the amount of root-exuded malic acid and chlorophyll fluorescence parameters. Note: $* *$ indicates significant difference at $p<0.001 ; *$ indicates significant difference at $p<0.005$. a: the value of Fv / Fm, b: the value of Fo, $\mathrm{x}$ : the amount of root-exuded malic acid.

\begin{tabular}{|c|c|c|c|c|}
\hline Plant species & $\begin{array}{l}\text { chlorophyll } \\
\text { fluorescence } \\
\text { parameters }\end{array}$ & Regression equation ${ }^{\#}$ & $\begin{array}{c}\text { Determination } \\
\text { coefficient } \\
\left(\mathrm{R}^{2}\right)\end{array}$ & $\begin{array}{c}\text { Number of } \\
\text { samples } \\
(\mathrm{N})\end{array}$ \\
\hline \multirow{2}{*}{ B.papyrifera } & $\mathrm{Fv} / \mathrm{Fm}$ & $\mathrm{a}=-10.869 \mathrm{x}+8.4284^{* *}$ & 0.9908 & \multirow{2}{*}{15} \\
\hline & Fo & $\mathrm{b}=0.0239 \mathrm{x}+0.0995^{* *}$ & 0.9563 & \\
\hline \multirow{2}{*}{ M. alba } & $\mathrm{Fv} / \mathrm{Fm}$ & $\mathrm{a}=-1.863 \mathrm{x}+1.603^{*}$ & 0.8840 & \multirow{2}{*}{15} \\
\hline & Fo & $\mathrm{b}=0.0462 \mathrm{x}+0.0955^{*}$ & 0.7854 & \\
\hline \multirow{2}{*}{ O. violaceus } & $\mathrm{Fv} / \mathrm{Fm}$ & $\mathrm{a}=-4.8213 \mathrm{x}+4.4567^{* *}$ & 0.9827 & \multirow{2}{*}{15} \\
\hline & Fo & $\mathrm{b}=0.0797 \mathrm{x}+0.0311^{* *}$ & 0.9827 & \\
\hline \multirow{2}{*}{ B. napus } & $\mathrm{Fv} / \mathrm{Fm}$ & $\mathrm{a}=-6.2747 \mathrm{x}+5.3018^{* *}$ & 0.9916 & \multirow{2}{*}{15} \\
\hline & Fo & $\mathrm{b}=0.0436+0.0507 * *$ & 0.9582 & \\
\hline
\end{tabular}

\section{Discussion}

Root-exuded organic acids are the main mediators of Krebs cycle, these organic acids are associated with respiration (Jones, 1998). As chlorophyll fluorescence parameters correspond to the properties of the photosynthetic characteristics, chlorophyll fluorescence technology is an effective method for studying plant stress physiology (Krause and Weis, 1991; Bolhar-Nordenkampf et al., 1989; Sayed, 2003).

Study results showed that the amount root-exuded malic acid increased with the decreased of $\mathrm{P}$ content in all four plant species (Figure 1), which is consistent with the results of previous studies (Jones, 1998; Egle et al., 2003). Corrales et al., (2007) shown that higher organic acids concentrations were detected in the rhizosphere of P-efficient than of P-inefficient maize in sand culture, $\mathrm{P}$ efficiency seemed due to enhanced $\mathrm{P}$ acquisition rather than to an enhanced $\mathrm{P}$ use efficiency. These organic acids mobilized $\mathrm{P}$ from insoluble phosphoric compounds such as $\mathrm{Ca}-\mathrm{P}$ and Fe-P to overcome $\mathrm{P}$ deficiency in calcareous soils and acid mineral soils. Therefore, B. papyrifera and $O$. violaceus was convenient to obtain a great amount of available $\mathrm{P}$ under $\mathrm{P}$ deficiency from rhizosphere soil via complexing with root-exuded organic acids.

The chlorophyll fluorescence parameters had significant difference in $\mathrm{P}$ supply and $\mathrm{P}$ deficiency. $P$ deficiency treatment made the maximum quantum yield of PSII, the maximum chlorophyll fluorescence decrease and the minimum chlorophyll fluorescence increase in Maize (Zea mays L. ) leaves (Li et al., 2004) and citrus leaves (Guo et al., 2002). Similar results (the minimum chlorophyll fluorescence increased 
and the maximum quantum yield of PSII decreased) presented in the four experimental plants (Figure 2). P deficiency had small destroy in PS II reaction centers in B. papyrifera and O. violaceus unlike in M.alba and B.napus. This conclusion is similar to the variation of the net photosynthetic rate: B. papyrifera is higher than M.alba (Wu et al., 2009), and O.violaceus is higher than B. napus (Wu et al., 2007). This finding indicates that B.papyrifera and O.violaceus have a stronger adaptability to a low-P environment than M.alba and B.napus.

As shown in Table 1, a linear equation can present the relationship between root-exuded malic acid and $\mathrm{Fv} /$ Fm, Fo. Photosynthesis and respiratory metabolism adaptability to a low-P environment occurred in B.papyrifera and O.violaceus. Therefore, the amount of root-exuded malic acid from plants at different $\mathrm{P}$ levels should be determined. This study was successful in presenting a low-cost methodology for assessing plant adaptability to a low-P environment in the absence of a chlorophyll fluorescence instrument.

\section{Conclusions}

Increasing in root-exuded malic acid was important mechanisms in response to $\mathrm{P}$ deficiency, while chlorophyll fluorescence parameters responded the degree of P stress deficiency. Compared with M. alba (B. napus), in response to $\mathrm{P}$ deficiency, the root of B. papyrifera (O. violaceus) actively released great amount of malic acid, while the maximum quantum yield of PSII and the minimum chlorophyll fluorescence decreased and increased significantly, respectively. A linear correlation between the amount of root-exuded malic acid and the minimum chlorophyll fluorescence (the maximum quantum yield of photosystem II) was accordingly obtained. Therefore, a low-cost method can be alternatively evaluated the plant adaptability to a low-phosphorus environment when no chlorophyll fluorescence instrument was available.

\section{Acknowledgements}

The authors gratefully acknowledge the financial support of the National Natural Sciences Foundation of China (No. 31070365), the "Strategic Priority Research Program - Climate Change: Carbon Budget and Related Issues" of the Chinese Academy of Sciences (Grant No. XDA05070400), A Project Funded by the Priority Academic Program Development of Jiangsu Higher Education Institutions, and the Project Funded by Graduate Innovative Cultivation of Jiangsu University (NO. CXLX11-0596), as well as the valuable comments and suggestions of the anonymous reviewers.

\section{References}

Bolhar-Nordenkampf, H. R., Long, S. P., Baker, N.R., Oquist, G., Schreiber, U., Lechner, E.G. 1989. Chlorophyll fluorescence as a probe of the photosynthetic competence of leaves in the field: a review of current instrumentation. Funct. Ecol. $3,497-514$.

Broadley, M. R., Burns, A., Burns, I.G. 2002. Relationships between phosphorus forms and plant growth. J. Plant. Nutr. 25, 1075-1088.

Cawthray, G.R. 2003. An improved reversed-phase liquid chromatographic method for the analysis of low-molecular mass organic acids in plant root exudates. J. Chromatogr. A. 1011, 233-240.

Corrales, I., Amenos, M., Poschenrieder, C., Barcelo, J. 2007. Phosphorus efficiency and root exudates in two contrasting tropical maize varieties. J. Plant. Nutr. 30, 887-900.

Dakora, F.D., Phillips, D.A. 2002. Root exudates as mediators of mineral acquisition in low-nutrient environments. Plant Soil. 245, 35-47. 
Egle, K., Romer, W., Keller, H. 2003. Exudation of low molecular weight organic acids by Lupinus albus L., Lupinus angustifolius L. and Lupinus luteus L. as affected by phosphorus supply. Agronomie. 23, 511-518.

Fu, W. G., Li, P. P., Wu, Y. Y. 2012. Effects of different light intensities on chlorophyll fluorescence characteristics and yield in lettuce. Sci. Hortic.Amesterdam 135, 45-51.

Guo, Y. P., Chen, P. Z., Zhang, L. C., Zhang, S. L. 2002. Effects of different phosphorus nutrition levels on photosynthesis in satsuma mandarin (Citrus unshiu Marc) leaves. Plant Nutr. Fertilizer Sci. 8, 186-191.

Hoagland, D. R., Arnon, D. I. 1950. The water-culture method for growing plants without soil. California Agricultural Experiment Station 347, 36-39.

Hoffland, E., Wei, C., Wissuwa, M. 2006. Organic anion exudation by lowland rice Oryza sativa L. at zinc and phosphorus deficiency. Plant Soil 283, 155-162.

Jones, D. L. 1998. Organic acids in the rhizosphere-a critical review. Plant Soil. 205, 25-44.

Krause, G. H., Weis, E. 1991. Chlorophyll fluorescence and photosynthesis: the basics. Annu. Rev. Plant Biol. Plant Mol. Biol. 42, 313-349.

Li, S. C., Hu, C. H., Gong, J., Dong, S. T. ,Dong, Z. X. 2004. Effects of low phosphorus stress on the chlorophyll fluorescence of different phosphorus use efficient Maize ( Zea mays L. ). Acta Agronomica Sinica. 30, 365-370.

Lippemeier, S., Frampton, D.M.F., Blackburn, S.I., Geier, S.C., Negri, A.P. 2003. Influence of phosphorus limitation on toxicity and photosynthesis of Alexandrium minutum (Dinophyceae) monitored by in-line detection of variable chlorophyll fluorescence. J. Phycol. 39, 320-331.
Lu, C. M., Zhang, J. H. 2000. Photosynthetic $\mathrm{CO}_{2}$ assimilation, chlorophyll fluorescence and photo inhibition as affected by nitrogen deficiency in maize plants. Plant Sci. 151, 135-143.

Meroni, M., Rossini, M., Guanter, L., Alonso, L., Rascher, U., Colombo, R., Moreno, J. 2009. Remote sensing of solar-induced chlorophyll fluorescence: review of methods and applications. Remote Sens. Environ. 113, 2037-2051.

Ogren, E. 1990. Evaluation of chlorophyll fluorescence as a probe for drought stress in willow leaves. Plant Physiol. 93, 1280-1285.

Qiu, D. L., Liu, X. H. 2000. Effect of simulated acid rain on the chlorophyll-a fluorescence characteristic of longan (Dimorcarpus longana Lour.) leaves. Acta Horticulturae Sinica. 27, 177-181.

Sayed, O.H. 2003. Chlorophyll flurescence as a tool in cereal crop research. Photosynthetica. 41, 321330 .

Wang, P., Zhou, R., Cheng, J.J., Bi, S.P. 2007. L.C determination of trace short-chain organic acids in wheat root exudates under aluminum stress. Chromatographia. 66, 867-872.

Wu, Y. Y., Li, P. P., Zhao, Y. G., Wang, J. Z., Wu, X. M. 2007. Study on photosynthetic characteristics of Orychophragmus violaceus related to shadetolerance. Sci. Hortic.-Amesterdam. 113, 173-176.

Wu, Y. Y., Liu, C. Q., Li, P. P., Wang, J. Z., Xing, D. K., Wang, B. L. 2009. Photosynthetic characteristics involved in adaptability to Karst soil and alien invasion of paper mulberry (Broussonetia papyrifera L.) Vent. in comparison with mulberry (Morus alba L.) . Photosynthetica. 47, 155-160. 